\title{
Latitudinal Structure of the Photospheric Magnetic Field through solar cycles
}

\author{
Elena Gavryuseva \\ Istituto Nazionale di Astrofisica - Arcetri Astrophysical Observatory, \\ Largo Enrico Fermi 5, I-50125 Florence, Italy \\ email: elena@arcetri.astro.it
}

\begin{abstract}
Latitudinal structure with four zones: two sub polar and two equatorial with 22-year period of polarity change is clearly demonstrated. The auto-correlation of the magnetic field has been calculated for all latitudes from $75 \mathrm{~N}$ to $75 \mathrm{~S}$ to study its temporal variability on a short and on a long term scale. The meridional drift of the magnetic field with $2-3$ years periodicities has been clearly evidenced in the both hemispheres.
\end{abstract}

Keywords. Sun: magnetic fields, photosphere, activity

The Wilcox Solar Observatory (WSO) data from http://wso.stanford.edu/synoptic. html used for the analysis are composed of 30 equal steps in latitude $\operatorname{sine}(\sin (\theta)$ from 75.2 North to 75.2 South degrees and of 5 degree steps in heliographic longitude in Carrington system covered the interval from 1976 to 2005.

Since the beginning of sunspots spectroscopic observations is known that their magnetic field is following to the Hale's law. Polarities of the sub-polar zones in the both hemispheres are opposite; they change around the maxima of the activity cycles; before the reversion during odd (even) cycles the polarity in the northern sub-polar regions is positive (negative) resulting in a solar magnetic cycle of about 22 years.

The magnetic field involved in the active regions has a very high concentration. Because of this the SMF intensity (SMFI) is closely related to the solar activity and follows to the famous "Butterfly Diagram" of the sunspot distribution over heliographic latitudes through solar cycles. Any averaging does not change the general shape of the $\operatorname{SMFI}(\theta, t)$ distribution while it makes difference for a magnetic field mean which considers the sign of the polarity.

To study the SMF structure it is necessary to take into account the intensity and the polarity of the field. Let us call the SMF mean over one or several full rotations of the Sun as the mean latitudinal of the field. Stability of the latitudinal field polarity and its value over several rotations and at the neighboring latitudes should be considered as evidence of the non random character of the latitudinal field. If such field exhibits some clear structure then it is important for the understanding of the origin of the solar activity (Parker, 1988; Shrauner \& Scherrer et al., 1994; Stenflo, 1994; Erofeev, 2001; Stix, 2004; etc.)

The search for long term latitudinal magnetic field structures should be performed by averaging the $\operatorname{SMF}(\theta, t)$ around the Sun at each latitude $\theta$ over one or more rotations. In the upper plot of Fig. 1 the mean over 1 Carrington rotation of the solar magnetic field is plotted as a function of time and latitude $\theta$. In the bottom plot the yearly running mean SMF with $1 \mathrm{CR}$ step is plotted. This averaging removes the yearly variability due to the orbital rotation of the Earth. The plots of Fig. 1 clearly show the existence of a global 4-zonal latitudinal structure in the stable over about 11 years having the following properties. 

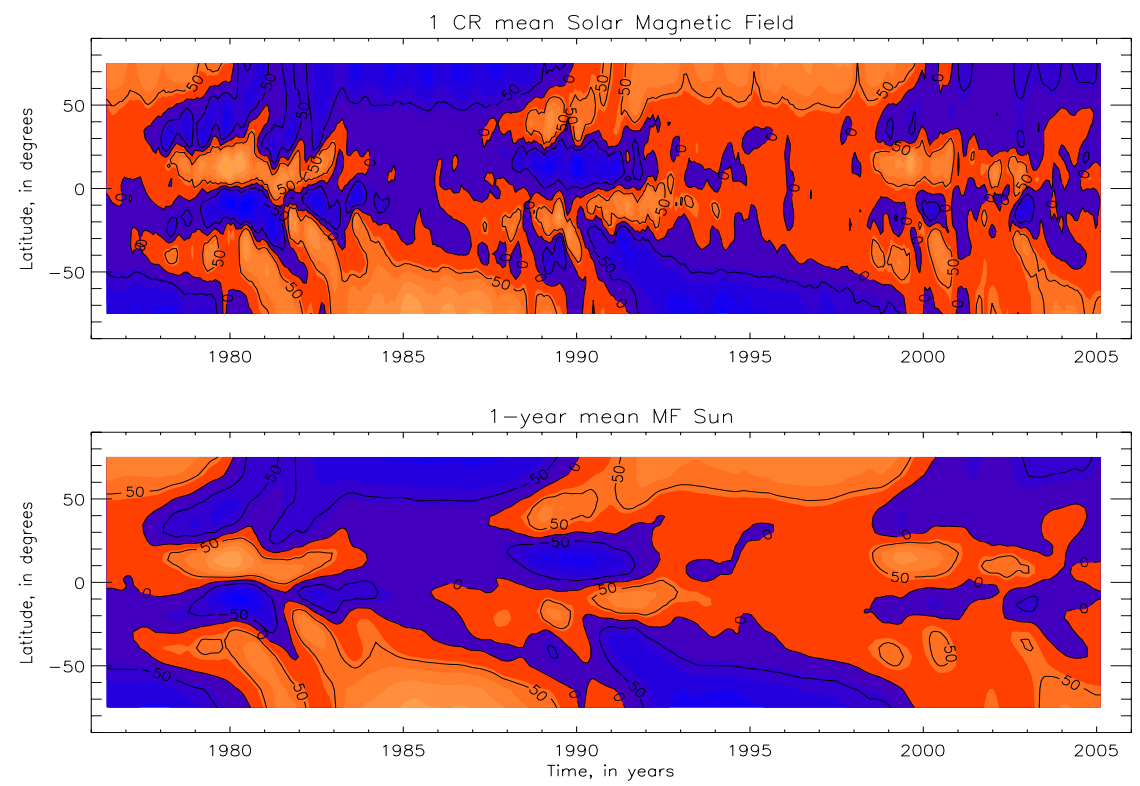

Figure 1. Distribution of the magnetic field in latitude and in time averaged over $1 \mathrm{CR}$ (upper plot) and over one year (bottom plot) with 1 CR step. Orange and blue (light and dark) colors indicate positive and negative polarities. The contours correspond to the levels of $-50,0,50$ micro Teslas.

(a) Four zones can be singled out: two pre-equatorial and two sub-polar with latitudinal boundaries $\theta$ equal to about $+25,0$ and -25 degrees.

(b) This large scale zonal structure is antisymmetric relative to the equator.

(c) The polarity is stable over about 11 years. The sub-polar zones change their polarity around the maximum of solar activity cycle often with some shift in time between the two hemispheres as seen in Fig. 1.

(d) The pre-equatorial zones have the same polarity as the leading part of most of the activity regions there; their polarities change from one cycle to the next $5-6$ years before the reversion in the sub-polar zones.

(e) The latitudinal structure is reconstructed with a periodicity of 18-22 years.

Let us call this SMF topology as 4-zonal 22-year periodical latitudinal structure (or shortly, $4 Z$-topology. This $4 Z$-topology is qualitatively described by the spherical function $Y_{l}^{m}(\theta, \phi)$, with spherical degree $l=3(l$ is the total number of nodal lines) and

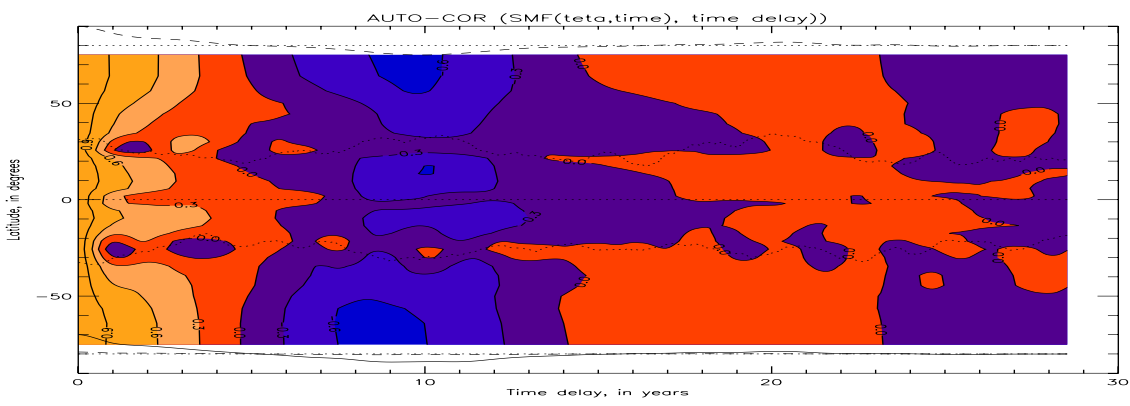

Figure 2. The auto-correlation of the 1-year mean SMF distribution as a function of time for different latitudes. 


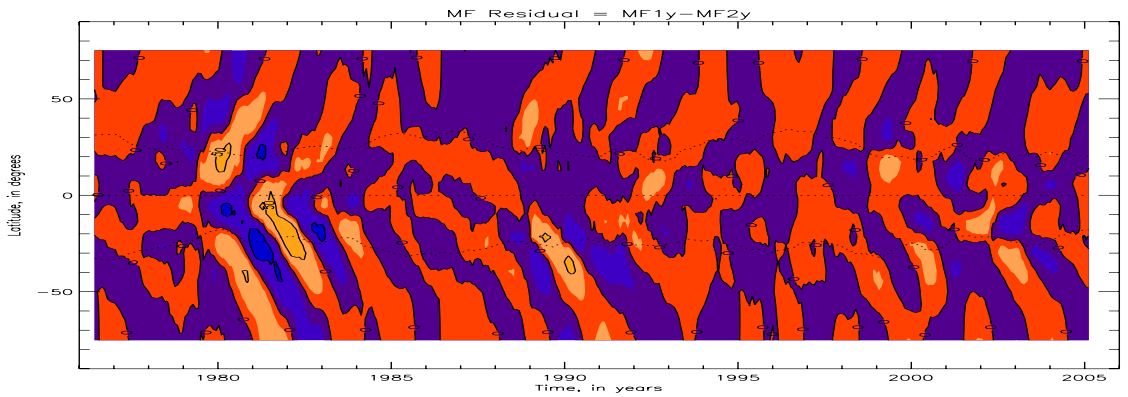

Figure 3. The yearly mean magnetic field after the filtering of the long term 4-zonal structure as a function of latitude and time with $1 \mathrm{CR}$ step.

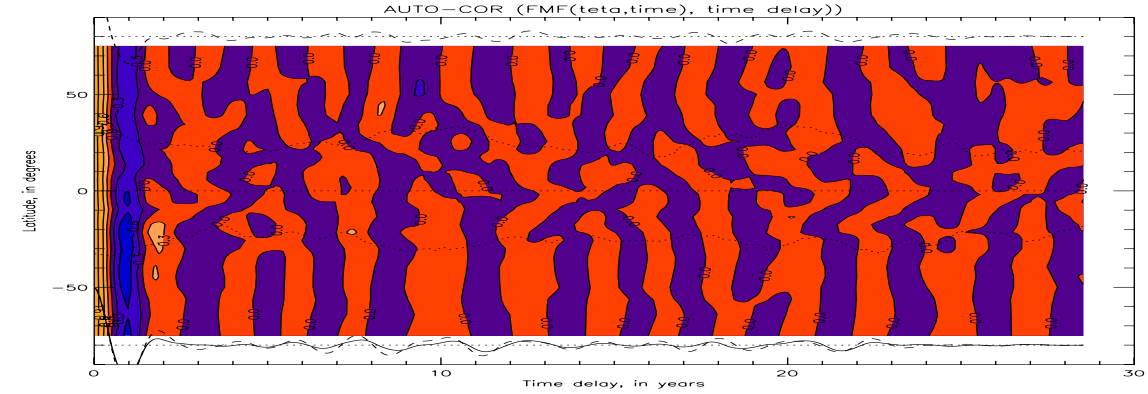

Figure 4. Coefficient of the auto-correlation of the filtered magnetic field FMF data sets as a function of time shift for each latitude.

azimuthal order $m=0$ ( $m$ is the number of nodal lines around the equator), modulated by the 22-year periodicity (Gavryuseva and Kroussanova, 2003).

The boundaries of the latitudinal zones are unexpectedly stable. They are very slowly approaching to the equator, significantly less than the drift of the SMFI maximum which shows the "Butterfly" migration. The amplitude of the 1 year mean solar line-of-sight magnetic field variability (MSMF) is 135-170 micro Teslas in the sub-polar zones at the latitudes of \pm 75 degrees. The amplitude in the near-equatorial zones is maximal at $10-15$ degrees latitudes and it is equal to 150-200 micro Teslas.

A quasi 20-year periodicity of the auto-correlation of the MSMF distribution for different latitudes as a function of time shift in years is illustrated by Fig. 2. Orange and blue (light and dark) colors correspond to the positive and negative coefficients of the $\operatorname{MSMF}(\theta, t)$ auto-correlation. An important characteristic of this auto-correlation is its very low level at the active latitudes, around zonal boundaries where the amplitude of the variability of the SMF intensity is the highest, and shorter period in near-equatorial zones ( 18-20 years) in comparison to the high latitude zones (20-22 years).

The four zonal quasi-stable structure could cover sub-structures of lower amplitude if they exist. To search them it is necessary to apply a filter to suppress the $4 Z$-structure as much as possible. Doing this an additional latitudinal topology of the SMF with the polarity stable over about one year about and running through the latitudes to the poles can be easily revealed. First Howard and LaBonte (1981) described discrete poleward streams. Then the axisymmetric variability was studied by Benevolenskaya (1996), and its relationship with solar activity was investigated by Erofeev (2001). Detail study of the running waves is desirable (Gavryuseva, 2006, 2006a). This dynamical topology with 
$1 y_{i} T_{j} 3 y$ is clearly visible in Fig. 3 where the latitudinal distribution of the SMF is presented after the filtering of long term 4-zonal structure from yearly mean SMF.

(a) The main difference from the $4 Z$ - zonal structure stable over 10-11 years discussed in the previous subsection is the running wave character of the polarity which is the same for all longitudes over one year and which is moving through latitudes.

(b) The amplitude of the $W R L$-deviation is of the order of 40 micro Teslas but it reaches 90 micro Teslas at the latitudes of 20-25 degrees South. This value is half of that in the $4 Z$-topology.

(c) These polarity running waves have a period of 2-3 years about, and they need 2.5-3 years to run from the equator to the poles with a velocity of about $40 \mathrm{~km} / \mathrm{h}$.

The presence of such polarity waves could explain the "double" maximum of the activity cycle. Some of the waves could be partly masked by the interference with the polarity waves turning back from the poles (sometimes visible as a wave running from one pole to the opposite one, seen in Fig. 3 in 1980-1990th).

The periodicity of the drifting latitudinal structure could be studied by the autocorrelation of the FMF data sets as a function of time shift for each latitude. The result of the calculation of the correlation coefficient is plotted in Fig. 4. Quasi 2-year periodicity is clearly visible. The mean correlations over all latitudes in each hemisphere are shown at the \pm 80 degrees levels (multiplied by factor 30 ). The high auto-correlation FMF and phase reconstruction over whole Sun takes place 20-21 years later. The FMF $W R L$-topology has a quasi 20-year periodicity.

(a) The latitudinal structure of the solar magnetic field with a polarity period of 22 years consists of four zones: two high latitude and two near-equatorial zones with boundaries around $+25,0$ and -25 degrees.

(b) The presence of polarity waves running from the equator to the poles with 2-3 years period during the last three cycles has been clearly demonstrated.

\section{Acknowledgements}

I thank very much the WSO team for the opportunity to use their data.

\section{References}

Benevolenskaya, E.E. 1996, Solar Phys., 167, 47.

Erofeev, D.V. 2001, Solar Phys., 198, 31.

Gavryuseva, E., \& Godoli, G. 2006, Physics and Chemistry of the Earth, Elsevier, 31, 68.

Gavryuseva, E. 2006a, News of the Academy of Sciences, Izv. TAN, Ser. Physics, 70, No.1, 102.

Gavryuseva, E., \& Kroussanova, N. 2003, Proc. of the Tenth International Solar Wind Conference, AIP Conf. Proc., 679, 242.

Howard, R.A., \& LaBonte, B.J. 1981, Solar Phys., 74, 131.

Parker, E.N. 1988, Solar. Phys., 110, 11.

Scherrer, P.H., Wilcox, J.M., Svalgaard, L., Duvall, T.L., Dittmer, Ph.H. \& Gustafson, E.K. 1977, Solar Phys., 54, 353.

Stenflo, J.O., Gudel, M. 1988, Astron.Astrophys., 191, 137.

Stix, M. 2004, The Sun. An Introduction, Second Edition, Springer-Verlag, Berlin. 